\title{
Parameters Affecting the Maximum Temperature of the Medium Voltage Cable Termination
}

\author{
Osama E. Gouda ${ }^{1}$, Adel Z. El Dein ${ }^{2}$, and H. M. Moneer ${ }^{2}$ \\ ${ }^{1}$ Faculty of Engineering, Cairo University, Egypt \\ ${ }^{2}$ Faculty of Energy Engineering, Aswan University
}

\begin{abstract}
The temperature distribution along the length of the termination of a single core cable as well as the maximum temperature location are calculated using an analytical method. Also, parameters affecting the maximum temperature along the length the medium voltage cable termination such as: the ambient temperature, the cable load current, insulation thermal conductivity, and termination dimensions (outer jacket length, length of the semi-conductor, length of the field regulator, thickness of the insulation, and thickness of the jacket) are discussed. The analytical method used is based on the heat flow produced by cable conductor and heat transfer by conduction, convection, and radiation.
\end{abstract}

\section{INTRODUCTION}

Power cables has been widely used in the transmission and distribution network. It has more advantages than overhead lines because excellent electrical and mechanical properties, saves the space, does not affect appearance of a city and does not interfere with communication lines. The length of power cable manufactured is limited (hundreds of meters) and due to this limitation of cable section to a certain length cable accessory (joints, terminations) must be used to connect cables of different parts. In recent years, medium voltage power cable has been widely used in the transmission and distribution network. However, the fault rate of cable accessories has increased year by year. During the fabrication and operation of cable accessories, the moisture can easily enter into cable accessories and make it damp, which will influence the insulation condition and lead to the failure of cable [1]. The highest conductor temperature in a cable system often occurs within a cable accessory rather than in a straight cable run. It is therefore important to study temperatures in cable accessories, both with full-size experiments and using computer models [2]. One of the dominant factors of cable termination fault in medium voltage circuit breaker compartment is due to excessive heating phenomenon. The excessive high temperature area will lead to degradation of the cable insulation. This eventually will lead to insulation failure and hence cable breakdown. The insulation failure may subsequently and causes fire in the cable compartment [3].

The objective of the paper is to estimate the temperature distribution along the medium voltage cable terminations for determining the maximum temperature location. Also the effects of the ambient temperature, the cable load current, insulation thermal conductivity as well as termination dimensions such as: outer jacket length, length of the semiconductor, length of the field regulator, thickness of the insulation, and thickness of the jacket, on the maximum temperature rise along the termination are investegated. During this study an electro-thermal analytical analysis is used [4] and validated by comparing its results with those obtained by experimental and finite element method.

\section{EleCtRo-ThERMAL ANALYSIS}

\section{Termination description}

In this paper the termination and the adjacent part of the cable are divided into eleven parts as given in Fig. 1. Table 1 presents the contents of each part as well as its length.

\section{Analytical method}

To explain the analytical used method the equivalent electrothermal circuit of the cable termination with neglecting the dielectric losses, is as shown in Fig. 2, in this figure the axial heat flow is considered.

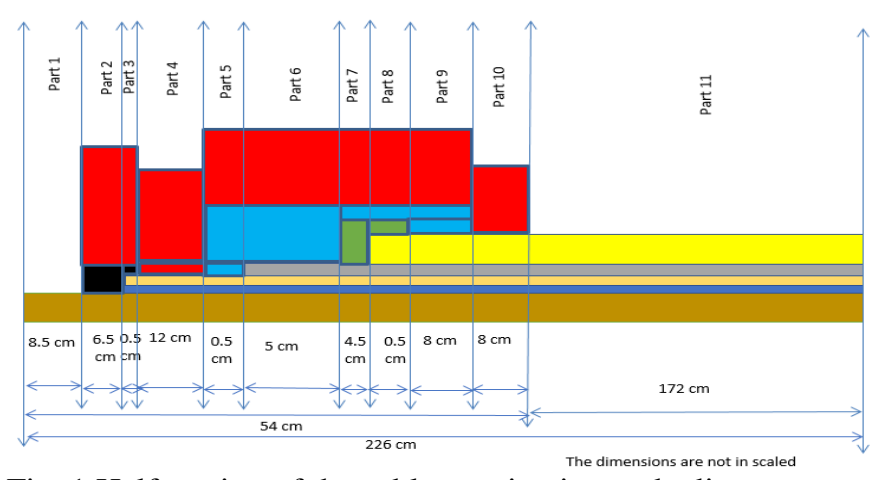

Fig. 1 Half section of the cable termination and adjacent parts

For a cable termination conductor element $d x$, the heat balance equation is as follows:

$\frac{d w}{d x}=w_{1}-w_{c}$

Where $w_{l}$ is the heat flow through the thermal resistance of the cable termination insulation to the ambient, and $w_{c}$ is the heat generated due to conductor losses, which is obtained by using equation (2) according to IEC60228 [5]: 


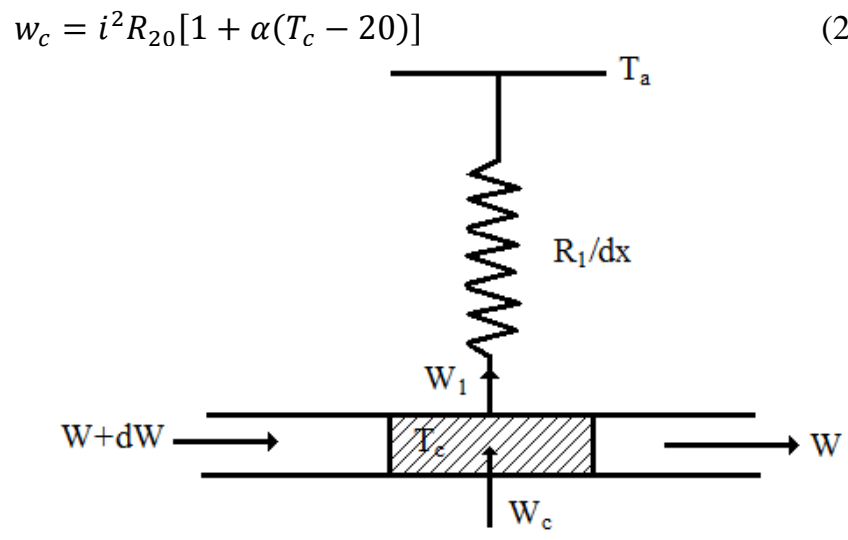

Fig. 2 Equivalent electro- thermal circuit of cable termination

Table 4.1 Each cable termination part length and components

\begin{tabular}{|c|c|c|c|}
\hline No. & Symbol & Contents & Length \\
\hline Part 1 & $F$ & Conductor & $8.5 \mathrm{~cm}$ \\
\hline Part 2 & $H$ & $\begin{array}{l}\text { Conductor, semi-conductor } \\
\text { and outer jacket }\end{array}$ & $6.5 \mathrm{~cm}$ \\
\hline Part 3 & $K$ & $\begin{array}{l}\text { Conductor, conductor screen, } \\
\text { insulation, semi-conductor } \\
\text { and outer jacket }\end{array}$ & $0.5 \mathrm{~cm}$ \\
\hline Part 4 & $L$ & $\begin{array}{l}\text { Conductor, conductor screen, } \\
\text { insulation and outer jacket }\end{array}$ & $12 \mathrm{~cm}$ \\
\hline Part 5 & $C$ & $\begin{array}{l}\text { Conductor, conductor screen, } \\
\text { insulation, field regulator and } \\
\text { outer jacket }\end{array}$ & $0.5 \mathrm{~cm}$ \\
\hline Part 6 & $N$ & $\begin{array}{l}\text { Conductor, conductor screen, } \\
\text { insulation, insulation screen, } \\
\text { field regulator and outer } \\
\text { jacket }\end{array}$ & $5 \mathrm{~cm}$ \\
\hline Part 7 & $P$ & $\begin{array}{l}\text { Conductor, conductor screen, } \\
\text { insulation, insulation screen, } \\
\text { plastic tape, field regulator } \\
\text { and outer jacket }\end{array}$ & $4.5 \mathrm{~cm}$ \\
\hline Part 8 & $\boldsymbol{R}$ & $\begin{array}{l}\text { Conductor, conductor screen, } \\
\text { insulation, insulation screen, } \\
\text { metallic copper, plastic tape, } \\
\text { field regulator and outer } \\
\text { jacket }\end{array}$ & $0.5 \mathrm{~cm}$ \\
\hline Part 9 & $x$ & $\begin{array}{l}\text { Conductor, conductor screen, } \\
\text { insulation, insulation screen, } \\
\text { metallic copper, field } \\
\text { regulator and outer jacket }\end{array}$ & $8 \mathrm{~cm}$ \\
\hline Part 10 & $Y$ & $\begin{array}{l}\text { Conductor, conductor screen, } \\
\text { insulation, insulation screen, } \\
\text { metallic copper and outer } \\
\text { jacket }\end{array}$ & $8 \mathrm{~cm}$ \\
\hline Part 11 & $Z$ & $\begin{array}{l}\text { Conductor, conductor screen, } \\
\text { insulation, insulation screen } \\
\text { and metallic copper }\end{array}$ & $172 \mathrm{~cm}$ \\
\hline
\end{tabular}

Where $R_{20}$ is the conductor resistance at $20{ }^{\circ} \mathrm{C}, \alpha$ is the temperature coefficient of the cable conductor material, $T_{c}$ is the conductor temperature, $i$ is the load current of the cable and $w$ is the longitudinal heat flow in the conductor, which can be obtained from the following relation:
$w=\frac{A}{\rho_{t h, C}} \frac{d T_{c}}{d x}$

Where $\rho_{t h, C}$ indicates the conductor thermal resistivity, and $A$ indicates the cross sectional area of the conductor. From equations (1), (2) and (3), the following differential equation can be obtained

$M=-a_{2} \frac{d^{2} T_{c}}{d x^{2}}+a_{o} T_{c}$

Where

$M=T_{a}+w_{20}(1-20 \alpha) R_{1}$

$a_{2}=\frac{R_{1} A}{\rho_{t h c}}$

$a_{o}=1-\alpha w_{20} R_{1}$

Where $R_{I}$ is the equivalent thermal resistance of the termination insulation layers and the surrounding medium, $W_{20}$ is the conductor losses at $20{ }^{\circ} \mathrm{C}, T_{a}$ is the ambient temperature. In Fig. 2 and equations (4) and (5) the dielectric losses are ignored. Equation (4) is a non-homogenous second order differential equation, which may be solved for each part of the eleven parts of the cable termination and adjacent cable parts as explained in detailed in [4], [6].

\section{APPLICATION OF THE ANALYTICAL METHOD}

The analytical method is applied on a cable termination of 15 $\mathrm{kV}, 240 \mathrm{~mm}^{2}$, Aluminum conductor, PVC isolated and PVC sheathed. According to IEC60287 standards [7], its rating is $355 \mathrm{~A}$ in air, $240 \mathrm{~A}$ in duct and $310 \mathrm{~A}$ when it is laid in the ground at $0.8 \mathrm{~m}$ laying depth. Table 2 presents the thickness and thermal conductivity of each material in the cable termination.

Table 2 Thickness and the thermal conductivity of each material in the cable termination

\begin{tabular}{|c|c|c|c|}
\hline Color & Material & Thickness & $\begin{array}{c}\text { Thermal } \\
\text { conductivity }\end{array}$ \\
\hline$\square$ & Conductor & $8.75 \mathrm{~mm}$ & 203.5 \\
\hline & $\begin{array}{c}\text { Conductor } \\
\text { screen }\end{array}$ & $0.15 \mathrm{~mm}$ & 350 \\
\hline & Insulation & $3.4 \mathrm{~mm}$ & 0.2 \\
\hline$\square$ & Insulation screen & $0.17 \mathrm{~mm}$ & 350 \\
\hline$\square$ & Metallic copper & $1.5 \mathrm{~mm}$ & 221 \\
\hline & Outer jacket & $4 \mathrm{~mm}$ & 0.09 \\
\hline & Field regulator & $2 \mathrm{~mm}$ & 0.012 \\
\hline & Plastic tape & $0.30 \mathrm{~mm}$ & 0.01 \\
\hline & Semi-conductor & $0.06 \mathrm{~mm}$ & 150 \\
\hline
\end{tabular}

According to the IEC 60287 standard [5], the thermal resistance of any insulating layer between two co-axial cylinders of internal and external radii $r_{1}$ and $r_{2}$ respectively per unit length can be calculated by the following relation [7]:

$R_{d_{-} t h}=\frac{\rho_{t h}}{2 \pi} \ln \left(\frac{r_{2}}{r_{1}}\right)$

Where $\rho_{t h}$ is the thermal resistivity for each dielectric layer in ${ }^{\circ} \mathrm{C} . \mathrm{m} / \mathrm{w}$. Also, the ambient thermal resistance $R_{a_{-} t h}$ can be calculated using the following equation [8].

$R_{a_{-} t h}=\frac{R_{a_{\_} t h(r a d)} \times R_{a_{\_} t h(n . c)}}{R_{a_{-} t h(r a d)}+R_{a_{-} t h(n . c)}}$ 
Where, $R_{a_{-} t h(\mathrm{rad})}$ is the radiation resistance, and it is calculated according to the following relation:

$R_{a_{-} t h(\mathrm{rad})}=\frac{0.6173 \times 10^{7}}{d\left(T_{s}^{2}+T_{a}^{2}\right)\left(T_{S}+T_{a}\right)}$

Where, $d$ is the external diameter of cable termination in meters, $T_{s}$ and $T_{a}$ are the surface and ambient temperatures in Kelvin degrees, respectively and $R_{a_{-} t h(n . c)}$ is the natural convection resistance, which is calculated according to equation (11):

$R_{a_{-} t h(n . c)}=\frac{0.1259}{d\left(T_{s}-T_{a}\right)}$

The conductor losses are calculated taking the skin effect and the variation of the conductor temperature into consideration at different loadings of the cable termination and adjacent cable parts. In this study both the dielectric losses and sheath losses are neglected, as the analysis is applied for medium voltage termination.

\section{EXPERIMENTAL SET UP}

The termination is prepared with respect the manufacture manual. Six sensors (thermo-couples) are inserted along the cable termination and the adjacent cable part. The test arrangement is given in Fig.3.

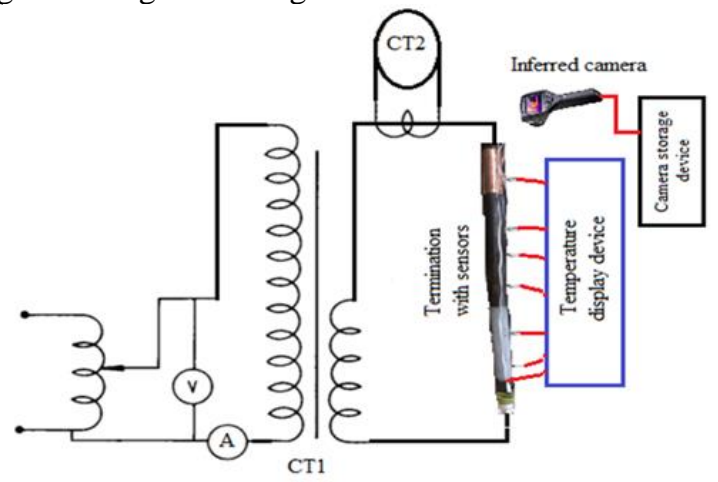

Fig. 3 Test arrangement

\section{Experimental Action}

Connecting the two terminals of $240 \mathrm{~mm}^{2}$ cable (with termination installed in it) with current source leads as shown in Fig. 3, then follow the following steps:

1) Adjust the temperature of the room (for ex ample at 22 degree) using air condition

2) Adjust loading current (for example at 180 amperes)

3) Adjust the output current of current source to 180 amperes.

4) Readings the sensors and record the conductor temperatures.

5) Record the temperature every 10 minutes until temperature dos not change (steady state).

\section{Finite Element Method}

In this paper, the COMSOL program package, which is based on the finite element method (FEM) [9-11], is used to simulate the case under study. As the case under study is symmetric around its axes, the model is treated as a two-dimensional heat flow problem. For any homogeneous region of a given thermal resistivity and heat generation rate, FEM can be used to obtain the temperature at any point in the region subjected to specified boundary conditions as explained in [4], [8].

\section{RESULTS AND DISCUSSION}

\section{Comparison Between the Obtained Results}

Fig. 4 shows comparison between the experimental measured steady state temperatures, results of the finite element method and analytical calculated temperatures distribution along the length of the termination at loading current $180 \mathrm{~A}$ and ambient temperature equals $22^{\circ} \mathrm{C}$. From this figure, it is noticed that there is a good agreement between the analytical results, finite element method results and the experimental measurements at the same conditions by using the fixed sensors.

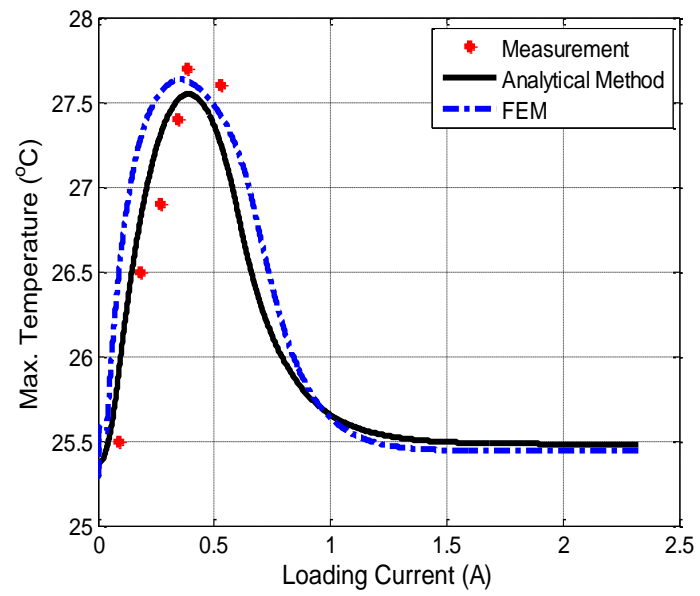

Fig. 4 Experimentally, FEM and analytically temperature distribution along the length of the termination at loading current equals $180 \mathrm{~A}$ and ambient temperature equals $22^{\circ} \mathrm{C}$

\section{Effect of the loading current}

In this part the effect of the loading current is studied at different ambient temperatures $\left(22^{\circ} \mathrm{C}, 26^{\circ} \mathrm{C}, 30^{\circ} \mathrm{C}\right.$, and $\left.35^{\circ} \mathrm{C}\right)$, as shown in Fig. Fig. 5. From this figure it is illustrated that the maximum temperature of the termination increases exponentially as the loading current is increased at fixed ambient temperature.

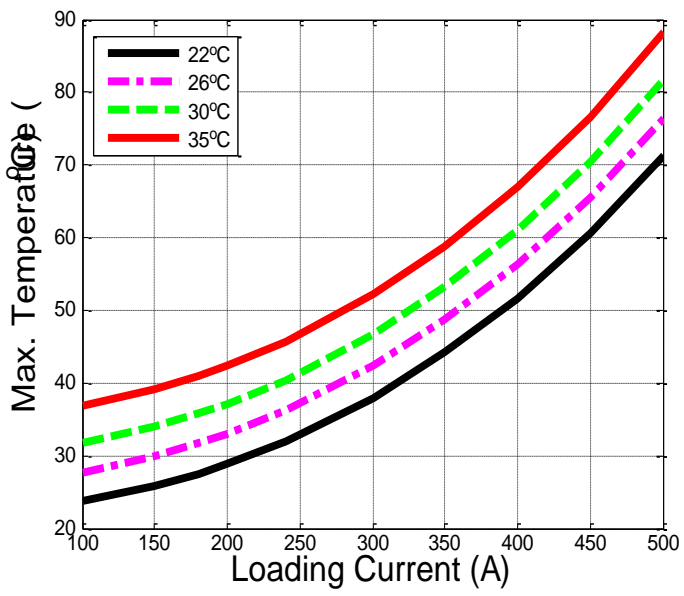

Fig. 5 Maximum termination temperature variation with the loading current at a constant ambient temperature 


\section{Effect of the ambient temperature}

In this part the effect of the ambient temperature on the maximum temperature of the termination is studied at a constant loading current equals $180 \mathrm{~A}$, and $240 \mathrm{~A}$, as presented in Fig. 6. Form the obtained results in this figure it is indicated that the surrounding temperature has significant effect on the termination maximum temperature.

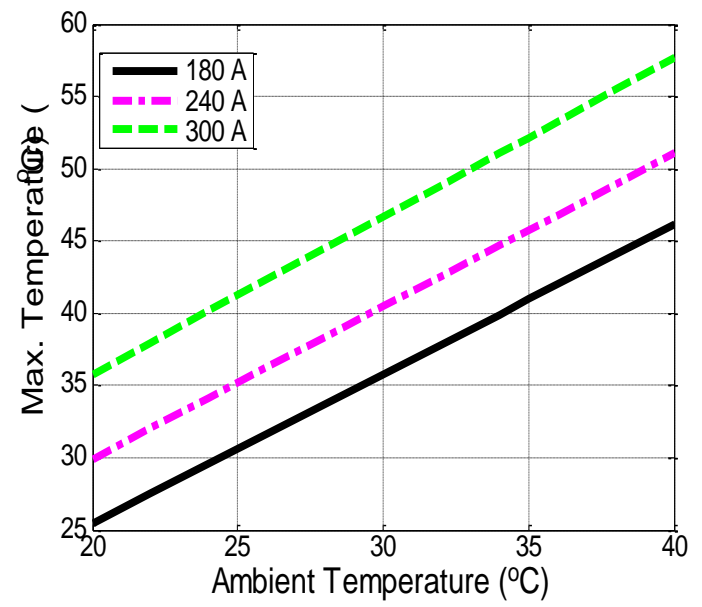

Fig. 6 Maximum termination temperature variation with the ambient temperature at a constant loading current

\section{Effect of the insulation thermal conductivity}

In this part the effect of the insulation thermal conductivity on the maximum temperature of the termination will be studied at a constant loading current and ambient temperature. To study the effect of the insulation thermal conductivity on the maximum temperature of the termination, the ambient temperatures take the value of $22{ }^{\circ} \mathrm{C}$ and the loading current equals 240A. Fig. 7 presents the variation of the maximum temperature of the termination with the variation of the insulation thermal conductivity. From the obtained results it is noticed that the maximum temperature of the termination decreases as the insulation thermal conductivity is increased. This is expected due to the corresponding decrease in the thermal resistance value of the insulating material with the increase in its thermal conductivity.

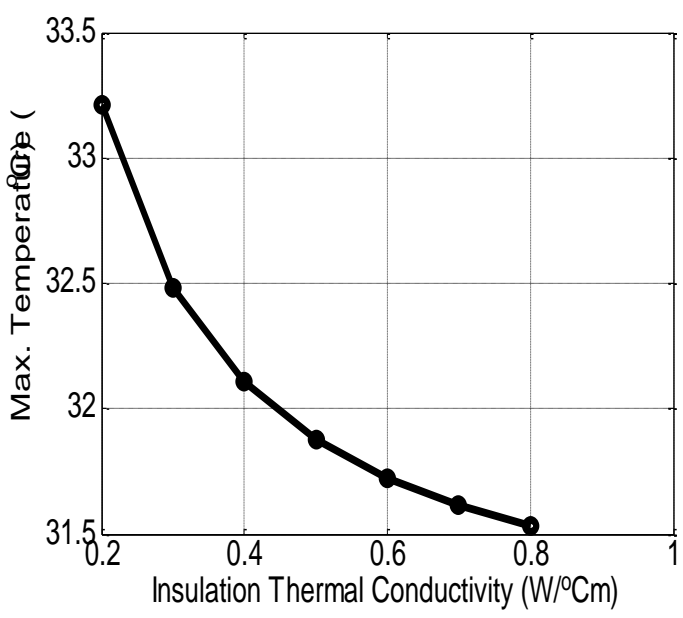

Fig. 7 Maximum termination temperature variation with the insulation thermal conductivity at a constant loading current $(240 \mathrm{~A})$ and a constant ambient temperature $\left(22^{\circ} \mathrm{C}\right)$

\section{Effect the termination dimensions}

\section{(a) Outer jacket length}

In this part the effect of the outer jacket length on the maximum temperature of the termination will be studied at a constant loading current and a constant ambient temperature. To study the effect of the outer jacket length on the maximum temperature of the termination, the ambient temperatures take the value of $22{ }^{\circ} \mathrm{C}$ and the loading current equals $240 \mathrm{~A}$.

Fig. 8 presents variation of the maximum temperature of the termination with the variation of the outer jacket length, at a constant loading current and a constant ambient temperature. From the obtained results in this part it is noticed that the maximum temperature of the termination increases as the insulation thermal conductivity is increased at a constant loading current and a constant ambient temperature.

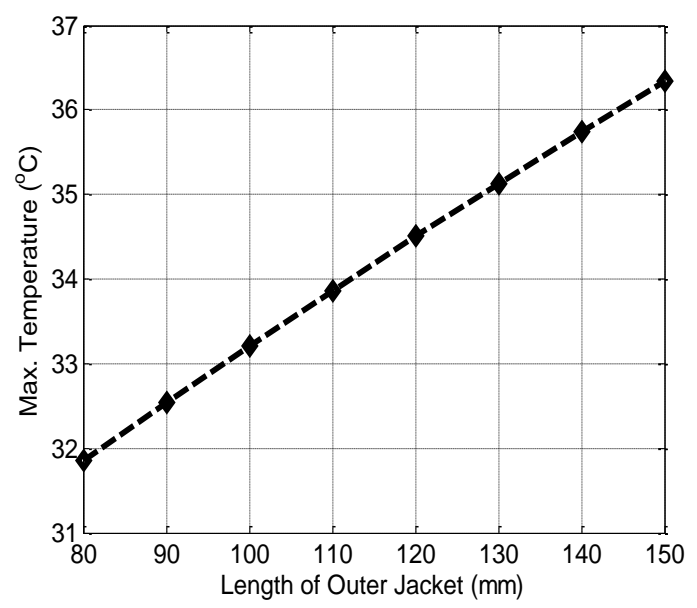

Fig. 8 Variation of the max. termination temperature with the variation of the Outer jacket length at a constant loading current $(240 \mathrm{~A})$ and a constant ambient temperature $\left(22^{\circ} \mathrm{C}\right)$

\section{(b) Length of the semi-conductor}

In this part the effect of the length of the semi-conductor on the maximum temperature of the termination will be studied at a constant loading current and a constant ambient temperature. To study the effect of the length of the semi-conductor on the maximum temperature of the termination, the ambient temperatures take the value of $22{ }^{\circ} \mathrm{C}$ and the loading current equals 240A. Fig. 9 presents variation of the maximum temperature of the termination with the variation of the length of the semi-conductor, at a constant loading current and a constant ambient temperature. From the obtained results in this part it is noticed that the maximum temperature of the termination increases as the length of the semi-conductor is increased at a constant loading current and a constant ambient temperature. 


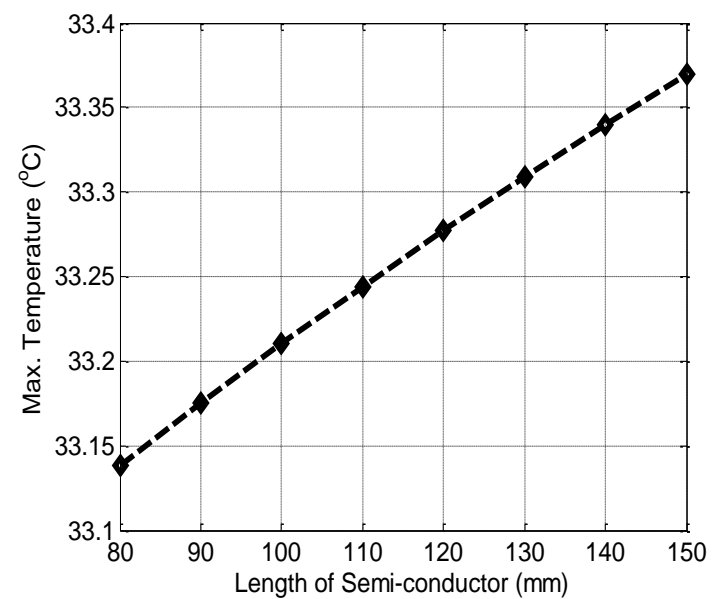

Fig. 9 Variation of the max. termination temperature with the variation of the length of the semi-conductor at a constant loading current $(240 \mathrm{~A})$ and a constant ambient temperature $\left(22^{\circ} \mathrm{C}\right)$

\section{(c) Length of the field regulator}

In this part the effect of the length of the field regulator on the maximum temperature of the termination will be studied at a constant loading current and a constant ambient temperature. To study the effect of the length of the field regulator on the maximum temperature of the termination, the ambient temperatures take the value of $22{ }^{\circ} \mathrm{C}$ and the loading current equals 240A. Fig. 10 presents variation of the maximum temperature of the termination with the variation of the length of the field regulator, at a constant loading current and a constant ambient temperature. From the obtained results in this part it is noticed that the maximum temperature of the termination increases as the length of the field regulator is increased at a constant loading current and a constant ambient temperature.

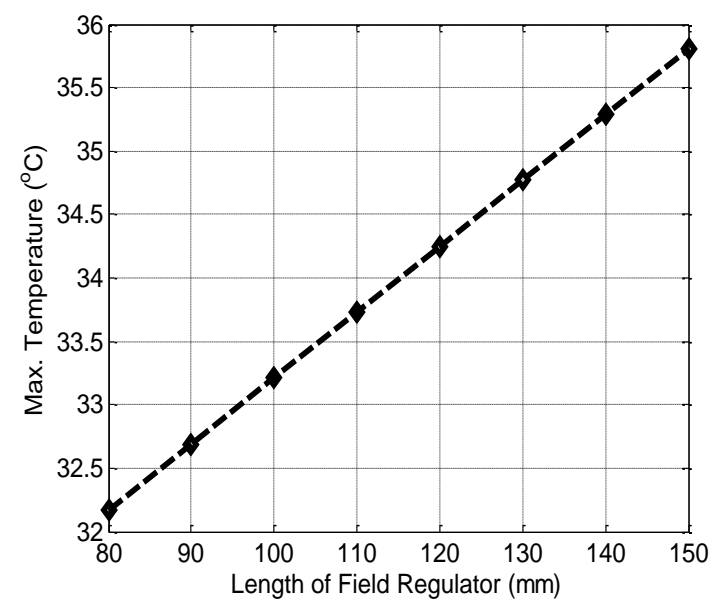

Fig. 10 Variation of the max. termination temperature with the variation of the length of the field regulator at a constant loading current (240A) and a constant ambient temperature $\left(22^{\circ} \mathrm{C}\right)$

\section{(d) Thickness of the insulation}

In this part the effect of the thickness of the insulation on the maximum temperature of the termination will be studied at a constant loading current and a constant ambient temperature. To study the effect of the thickness of the insulation on the maximum temperature of the termination, the ambient temperatures take the value of $22{ }^{\circ} \mathrm{C}$ and the loading current equals 240A. Fig. 11 presents variation of the maximum temperature of the termination with the variation of the thickness of the insulation, at a constant loading current and a constant ambient temperature. From the obtained results in this part it is noticed that the maximum temperature of the termination increases as the thickness of the insulation is increased at a constant loading current and a constant ambient temperature.

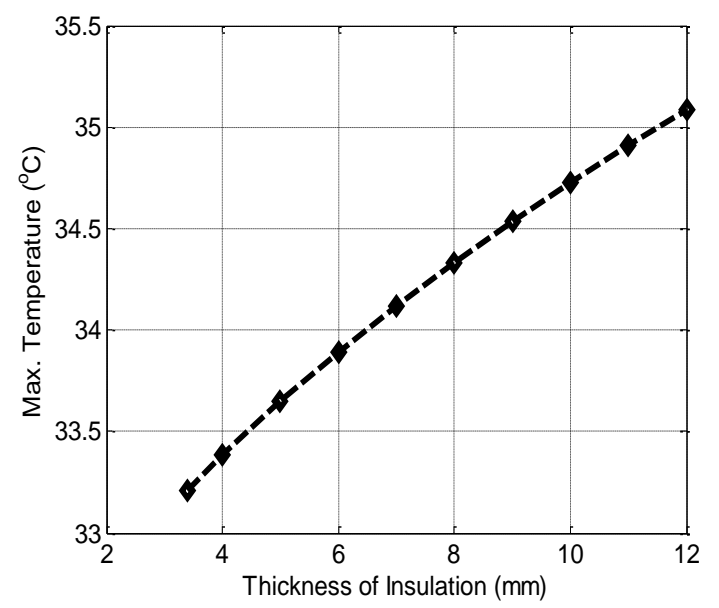

Fig. 11 Variation of the max. termination temperature with the variation of the thickness of the insulation at a constant loading current (240A) and a constant ambient temperature $\left(22^{\circ} \mathrm{C}\right)$

\section{(e) Thickness of the jacket}

In this part the effect of the thickness of the jacket on the maximum temperature of the termination will be studied at a constant loading current and a constant ambient temperature. To study the effect of the thickness of the jacket on the maximum temperature of the termination, the ambient temperatures take the value of $22{ }^{\circ} \mathrm{C}$ and the loading current equals 240A. Fig. 12 presents variation of the maximum temperature of the termination with the variation of the thickness of the jacket, at a constant loading current and a constant ambient temperature. From the obtained results in this part it is noticed that the maximum temperature of the termination increases as the thickness of the jacket is increased at a constant loading current and a constant ambient temperature. 


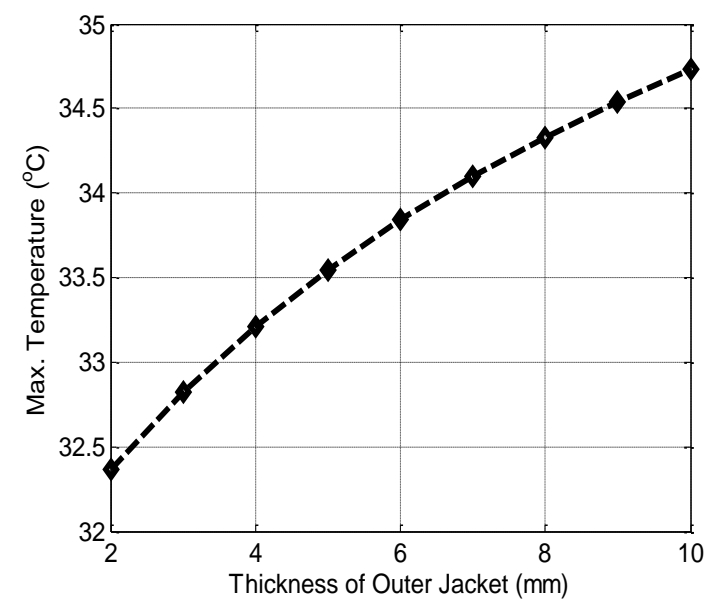

Fig. 12 Variation of the max. termination temperature with the variation of the thickness of the outer jacket at a constant loading current (240A) and a constant ambient temperature $\left(22^{\circ} \mathrm{C}\right)$

\section{CONCLUSIONS}

Also, the suggested electro-thermal analytical analysis has been applied to a termination of $15 \mathrm{kV}, 240 \mathrm{~mm} 2$ single core distribution cable. A comparison of the simulated temperature rises with actual measurements as well as with the finite element method results reveals a good agreement.

The present method can be applied for high voltage

terminations with including the dielectric losses but possessing the same general features.

\section{REFERENCES}

[1] Weiwei Li, Fang Xin, Ke Zhu, Bin Zhu, Ye Yuan, Zhidong Jia Mengxin Song, XiLin Wang, "Research on Insulation Condition of Damp Cable Termination under Different Temperatures", 2016 IEEE International Conference on Dielectrics (ICD), 3-7 July 2016.

[2] R.C. Broughton, A.J. Oliver, D.R. Soulsby, P.L. Stephenson, W. Boone, and J. Kuiper, "Investigation of temperature and oil flow in EHV Sealing ends", IEE PROCEEDINGS, Vol.136, Pt.C, No.4, JULY 1989, pp. 230- 237.

[3] Z.A. Abu Zarim, A.B. Abd Ghani, and S.K. Tiong, "The Measurement and Temperature Profile of Cable Insulation Failure due to Lose Connection at the Cable Termination", Proceedings of 2008 International Symposium on Electrical Insulating Materials, September 7-11, 2008, pp. 570-572, Yokkaichi, Mie, Japan

[4] Osama E. Gouda, Adel Z.El dein and H. M. Mounir "Electro-thermal Analysis of Natural cooling Extra High Voltage Cable Sealing end Provided with Heat pipe As cooling Method", 2016 Eighteenth International Middle East Power Systems Conference (MEPCON), Cairo, 27-29 Dec. 2016.

[5] IEC 60228, the International Electrotechnical Commission's international standard on conductors of insulated cables. The current version is Third Edition 2004-11

[6] Ossama E. Gouda, Adel Z. El Dein "Electro-Thermal Analysis of Low and Medium Voltage Cable Joints", Electric Power Components and Systems, Vol. 44, Iss.1, 2016, pp. 110-121.

[7] IEC: 'Calculation of the continuous current rating of cables (100\% load factor)'. International Electrotechnical Commission Standard 60287, 1982

[8] O. E. Gouda: 'Environmental Impacts on Underground Power Distribution', Advances in Computer and Electrical Engineering, IGI Global, 2016
[9] Gouda, O. E., El Dein, A. Z., and Amer, G. M., "Effect of the formation of the dry zone around underground power cables on their ratings," IEEE Trans. Power Del., Vol. 26, No. 2, pp.972-978, April 2011.

[10]Shaker, Y. O., El-Hag, A. H., Patel, U., and Jayaram, S. H., "Thermal modeling of medium voltage cable terminations under square pulses," IEEE Trans. Dielectric Electrical Insulation, Vol. 21, No. 3, pp. 932939, June 2014

[11]Lin, S., and Hu, W., "Theoretical research on temperature field of power cable joint with FEM," International Conference on System Science and Engineering, pp. 564-567, Dalian, China, 30 June-2 July 2012. 